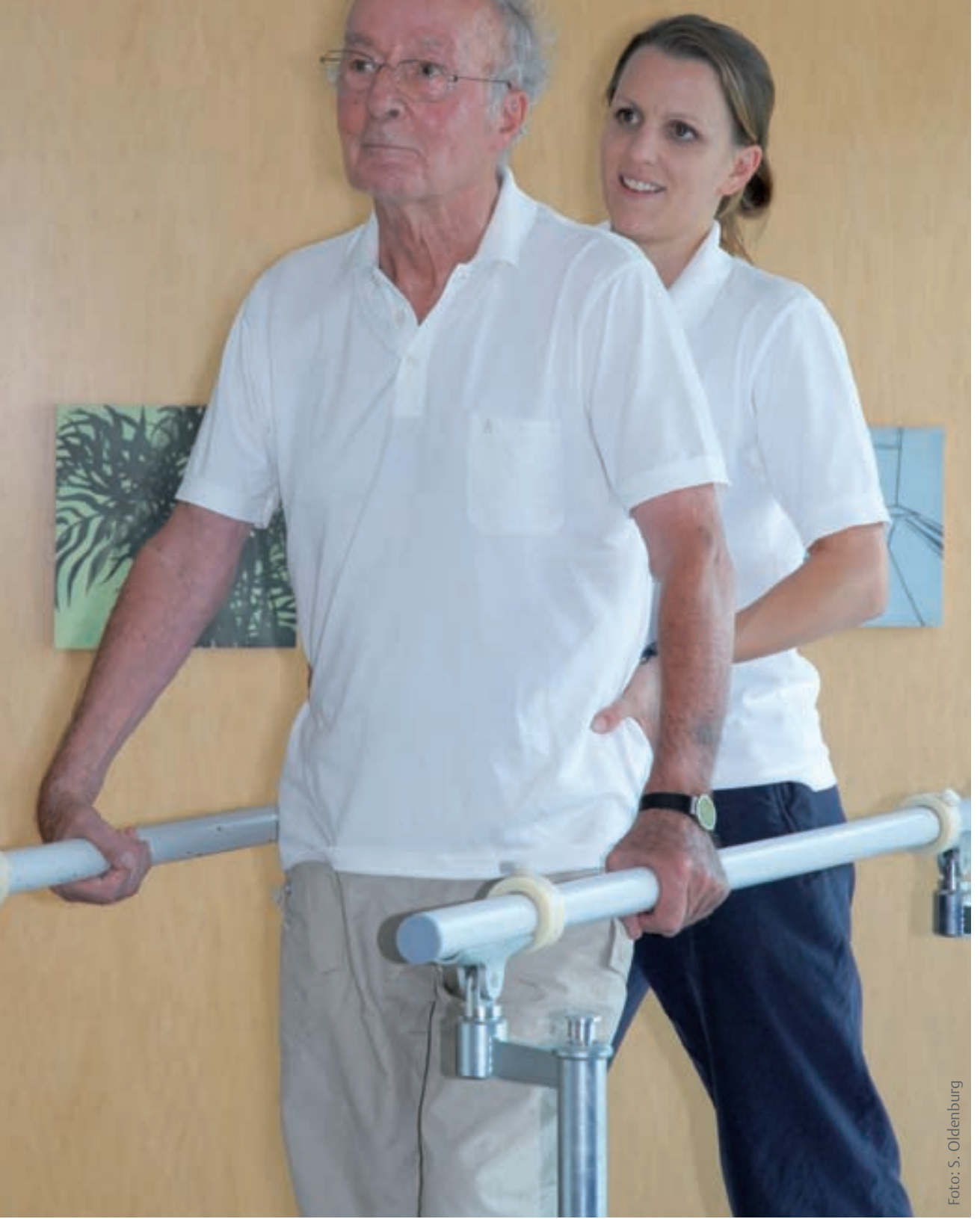

\section{Gehen und Fallangst}

Stürze ereignen sich vor allem bei Patienten nach Schlaganfall, bei Morbus Parkinson und bei vestibulären Störungen. Neben dem hohen Risiko von Frakturen führen sie bei den Betroffenen zu verminderter Aktivität und sozialem Rückzug, und damit zu einer Beeinträchtigung der Teilhabe. Die wesentlichen Ursachen für Stürze liegen in posturaler Instabilität und Einschränkungen der Gehfähigkeit, aber auch in der Fallangst. Zusätzlich korrelieren Fallangst und die Häufigkeit von Stürzen hoch mit der Diagnose Depression. Angst selbst vermindert posturale Stabilität. Die Erfahrung von Stürzen erhöht die Fallangst. Hier entsteht bei den Betroffenen rasch ein Circulus vitiosus. Diese Spirale der Verschlechterung wird zu einem schweren Hindernis für die Gehfähigkeit und Mobilität. Sturzprävention ist daher eine vorrangige therapeutische Aufgabe. Dieser Artikel stellt klar, wie wichtig ein interdisziplinärer Ansatz zur Reduktion der Fallangst ist.
Ingrid Scholler, Claudia Pott und Wolfgang Fries

\section{Einleitung}

Der aufrechte Gang des Menschen erzwingt vom Körper ein hohes Maß an posturaler Kontrolle. Für die Aufgabe, das Gleichgewicht zu wahren, müssen vestibuläre, visuelle und somatosensible Informationen aufgenommen und zentral im ZNS verarbeitet werden, um die muskuläre Aktivität zu steuern. Denn die Voraussetzung für einen sicheren Stand und Gang ist, dass der Massenschwerpunkt des Körpers über der Unterstützungsfläche gehalten wird. Dies stellt während des dynamischen Prozesses des Gehens eine große Herausforderung dar. Einschränkungen in der posturalen Stabilität, in neuronalen und/oder muskulären Funktionen oder die Erschwerung der physikalischen Bedingungen führen nicht nur zur Gefahr eines Sturzes. Diese Einschränkungen lösen bei den Betroffenen - berechtigterweise - auch ein Angstgefühl aus. Dies lehren bereits alltägliche Erfahrungen wie das Gehen mit einem „eingeschlafenen“ Bein (Gangunsicherheit bei Verlust der Tiefensensibilität) oder das Gehen über einen Schwebebalken (Angst und daraus resultierende Unsicherheit bei Erhöhung und Verkleinerung der Unterstützungsfläche).

Neurologische Erkrankungen und erworbene Hirnschädigungen beeinträchtigen entweder die sensorische Informationsaufnahme, die zentrale Verrechnung und/oder die muskuläre Steuerung. Sie schränken die Gleichgewichtsreaktionen und die Gehfähigkeit der Betroffenen ein und erhöhen das Risiko zu stürzen. Deshalb haben besonders Menschen nach Schlaganfall, ein hohes Risiko zu stürzen. In Interviews geben Patienten nach Schlaganfall Fallangst als „natürlichen“ Anteil in ihrem Alltagsleben an [33]. Denn ein Sturz birgt das Risiko von Verletzungen und führt zur Entwicklung von Fallangst. Beides führt zur Verminderung von Aktivität und Mobilität, zu sozialem Rückzug und unter Umständen zum Verlust von Unabhängigkeit [43], also Einschränkungen der Teilhabe. Umgekehrt erhöht Fallangst auch das Risiko zu stürzen [12].

\section{Inzidenz von Stürzen nach Schlaganfall}

Die meisten Daten zu Stürzen liegen für Patienten nach Schlaganfall vor. Weitere häufige Ursachen von Stürzen nach neurologischer Erkrankung sind die Parkinsonsche Erkrankung, periphere und zentrale vestibuläre Schädigungen sowie Demenz [12, 38]. 
Die Angaben zur Inzidenz von Stürzen nach Schlaganfall schwanken erheblich (zwischen 23\% und 67\%) und sind abhängig von der Art der Studie, der untersuchten Patientengruppe und der Chronizität. Nach Entlassung aus der stationären Rehabilitation berichten 67,3\% der befragten Patienten, die den Fragebogen zurücksandten, über einen oder mehrere Stürze [42]. In einer 6-Monats-Katamnese bei 1104 Patienten nach Schlaganfall in Neuseeland stürzten $37 \%$ der Patienten mindestens einmal [22]. In einer anderen Studie hatten - ebenfalls nach 6 Monaten - 50\% der Patienten einen Sturz erlitten [15]. In einer Langzeitstudie an Personen, die mit einer Chronizität bis zu 10 Jahren nach Schlaganfall in einem häuslichen Umfeld lebten, lag die Häufigkeit von Stürzen bei $23 \%$ der Befragten. Demgegenüber erlitten nur $11 \%$ der alters- und geschlechtsentsprechenden Kontrollpersonen ohne Schlaganfall einen Sturz [19]. Die wesentlichen Ursachen für Stürze liegen in posturaler Instabilität und Beeinträchtigungen der Gehfähigkeit, aber auch in der Fallangst [43]. Die Mehrzahl der Stürze ereignet sich innerhalb des Hauses [22].

\section{Faktoren, die zur Fallangst beitragen}

Als wesentliche Faktoren für das Entstehen von Fallangst nach einem Schlaganfall nennt die Mehrzahl der Studien: vorangegangene Stürze, die Wahrnehmung von körperlichen Einschränkungen des Gehens und der posturalen Kontrolle sowie die das tägliche Leben bestimmende Angst, erneut zu fallen [33].

Generell hat Angst eine wichtige Funktion. Als ein die Sinne schärfender Schutzmechanismus, der in tatsächlichen oder auch nur vermeintlichen Gefahrensituationen ein angemessenes Verhalten einleitet. Als Instrument zur Messung von Fallangst hat sich international die Falls Efficacy Scale (FES) durchgesetzt. Die FES wurde zunächst zur Erfassung der sturzassoziierten Selbstwirksamkeit entwickelt. In diesem Konzept geht es darum, wie viel Bedenken eine Person hat hinzufallen, wenn sie bestimmte Aktivitäten im und außer Haus ausführt. Dieses Konzept überlagert sich weitgehend mit dem der Fallangst, wie Jorstadt und Kollegen in einem systematischen Review zeigten [20]. Die Original-FES-Skala wurde im Verlauf modifiziert und eine internationale Version entwickelt, die Dias und Kollegen 2006 ins Deutsche übersetzten [7]. In dieser
Skala wird abgefragt, wie groß die Bedenken zu stürzen sind ( $\odot$ Kasten „Auszüge aus der Falls Efficacy Scale“).

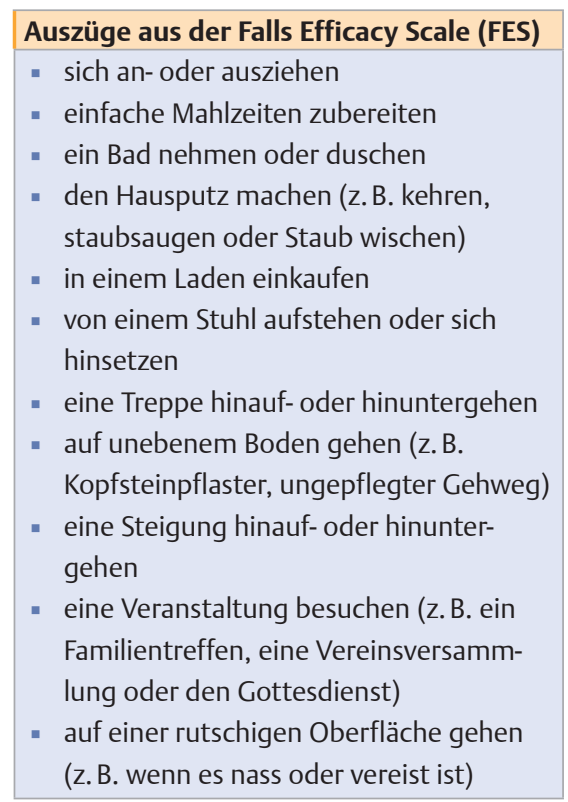

Fallangst nach Schlaganfall ist häufig. So berichten Watanabe und Mitarbeiter bei 59,2\% der befragten Schlaganfallpatienten über die Entwicklung einer Fallangst, nachdem sie gestürzt waren [42]. Die ursächli- chen Faktoren stehen dabei jedoch in einem wechselseitigen Zusammenhang. Ebenso wie Stürze ein unabhängiger Prädiktor für Fallangst sind, ist Fallangst selbst ein unabhängiger Prädiktor für Stürze [4, 12]. In einer Studie zeigten 20\% der Patienten eine erhöhte Fallangst, ohne gestürzt zu sein, und $11 \%$ derjenigen, die bereits gestürzt waren, zeigten keine Fallangst [2]. Im Ergebnis führen sowohl erlebte Stürze als auch erhöhte Fallangst zu einer Spirale erhöhten Risikos, tatsächlich zu fallen, ebenso wie zum Risiko, Fallangst zu entwickeln [12] (๑ Abb.1).

Als Ursachen für Fallangst nach Schlaganfall werden neben der Erfahrung von Stürzen vor allem auch posturale Instabilität und Beeinträchtigung der Gehfunktion diskutiert $[2,4,15,43]$. Untersuchungen an gesunden Probanden haben gezeigt, dass Fallangst, die experimentell durch Höhenunterschiede erzeugt wird, einen direkten Einfluss auf die posturale Kontrolle hat, posturale Stabilität kompromittiert $[1,6]$ und zu einer spinalen Reflexmodulation mit verminderter $\mathrm{H}-\mathrm{Re}$ flex-Amplitude führt [37].

Ein weiterer Zusammenhang mit Stürzen und Fallangst ist in Depressionen zu sehen. Es besteht ein enger Zusammenhang zwischen depressiven Symptomen und Stürzen

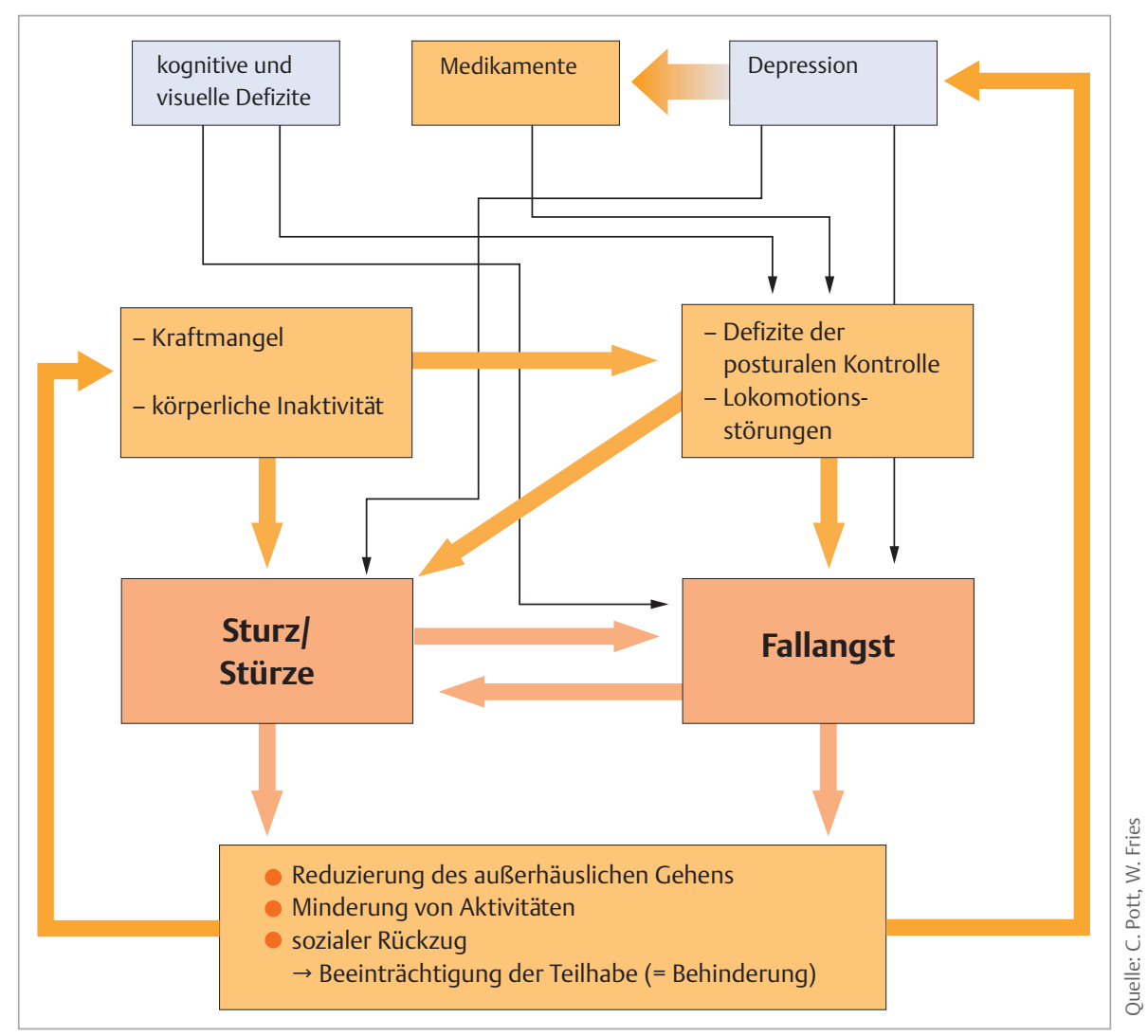

Abb. 1 Teufelskreis von Sturz/Stürzen und Fallangst 
nach Schlaganfall. Diese beiden Variablen korrelieren hoch [4, 22]. Das Ausmaß der depressiven Symptomatik erlaubt eine Vorhersage für das Risiko von Stürzen [19]. Andere kognitive Einschränkungen sowie visuelle Wahrnehmungsstörungen (wie z. B. Hemianopsie oder Neglekt) erhöhen das Sturzrisiko ebenfalls. Dabei sollte der Einfluss von Medikamenten nicht übersehen werden. Bei geriatrischen Patienten findet sich eine besonders hohe Korrelation zwischen Stürzen und der Einnahme von Medikamenten, vor allem Psychopharmaka, besonders bei trizyklischen Antidepressiva und Typ-Ia-Antiarrhythmika [34].

\section{Therapeutische Interventionen: Konzepte und praktische Vorgehensweise}

\section{Physiotherapeutische Behandlung}

Im Falle einer extern einwirkenden Kraft (angerempelt werden) oder bei Schwerpunktverlagerung über die Unterstützungsfläche hinaus, zum Beispiel beim Aufheben eines Gegenstandes vom Boden, droht der Verlust der posturalen Stabilität. Um diesen Verlust zu verhindern, erfolgen Kompensationsbewegungen oder Schutzschritte. Bei den Strategien zur Sturzvermeidung werden die Sprunggelenks-, Hüft- und Schrittstrategie unterschieden [17, 29]. Sind diese Mechanismen zur Fallverhinderung nicht ausreichend, stürzt man.

Die physiotherapeutische Intervention besteht aus Assessments und einem komplexen motorischen Training. Zu den Assessments gehören Tests zur Quantifizierung der sensomotorischen Störungen, wie Ausmaß der Parese, Spastik, Störungen der Sensibilität etc., die als Folge des „Upper-Motor-Neuron-Syndroms“ zu erwarten sind. Die Fähigkeiten der posturalen Kontrolle werden mit entsprechenden Testverfahren überprüft: der Berg Balance Scale [5], dem Geh-undZähl-Test [14] und dem Timed-Up-and-GoTest [31]. Bei positiver Sturzanamnese sollte die FES hinzugezogen werden. Die motorischen Maßnahmen, die physiotherapeutische Sturzprävention umfassen, sind:

- Krafttraining

- Geschwindigkeitstraining

- posturales Gleichgewichtstraining

- Terraintraining

- Sturztraining (Hilfsmittelversorgung und Hausbesuch)

Das physiotherapeutische Training soll die Kraft und posturale Kontrolle der Patienten verbessern und dadurch das Sturzrisiko vermindern. Ergänzt werden diese Trainingseinheiten durch eine optimale Hilfsmittelversorgung. Häufig entlassen stationäre Rehabilitationseinrichtungen die Patienten ohne Orthese oder den passenden Stock.

Im Rahmen eines Hausbesuchs identifiziert die Physiotherapeutin mögliche Sturzquellen und überprüft, inwieweit die erreichten Fähigkeiten der Lokomotion und posturalen Kontrolle in den individuellen Alltagskontext übertragen werden können.

\section{Krafttraining}

Bei Fallangst und zur Sturzprävention steht die Kräftigung der unteren Extremität im Setting von Einzel-, Gruppen- oder Selbsttraining im Vordergrund. Entsprechend der drei Strategien sollten besonders die sprunggelenksumgebende Muskulatur, der Quadrizeps und die ischiokrurale Muskulatur mit unterschiedlichen Methoden trainiert werden. Zur Wahl stehen funktionsorientiertes Üben, wie das Treppensteigen, oder Krafttraining gegen Widerstand. Widerstandstraining kann der Patient mithilfe von Gewichtsmanschetten oder an speziellen Trainingsgeräten, wie dem Motomed, absolvieren.

Auch im Fitnessstudio ist Krafttraining unter physiotherapeutischer Anleitung möglich. Dort kann gemeinsam mit dem Patienten und dem Trainer vor Ort ein geeignetes Krafttraining geplant werden. Eine Inklinationsmöglichkeit des Laufbandes verbessert die Trainingsmöglichkeiten für das Kraft- und Ausdauertraining. In der Einzeloder Gruppenintervention sollte das Treppensteigen fester Bestandteil zur Steigerung der kardiopulmonalen Fitness und der Kraft sein. Die zum Treppensteigen benötigte Kraft ist doppelt so hoch wie die benötigte Kraft zum Gehen in der Ebene. Treppen sind in der normalen Umgebung ausreichend vorhanden und können deshalb häufig und wiederholt zum Üben genutzt werden.

\section{Geschwindigkeitstraining}

Die selbst gewählte Ganggeschwindigkeit ist ein Indikator für das Vertrauen in die eigenen Lokomotionsfähigkeiten [35]. Der Zusammenhang zwischen Ganggeschwindigkeit und der Angst zu stürzen wurde oben ausführlich dargestellt. Therapeuten müssen daher das Ziel fokussieren, die selbst gewählte und maximale Geschwindigkeit zu erhöhen. Ein strukturiertes Geschwin- digkeitstraining auf dem Laufband hat sich als wirksam zur Verbesserung der Ganggeschwindigkeit erwiesen [32]. Dabei wird die Geschwindigkeit des Laufbandes sukzessive so lange gesteigert, wie es vom Patienten toleriert wird und kein Stolpern auftritt. Zusätzlich sollte ein spezifisches Geschwindigkeitstraining im Alltagskontext stattfinden.

\section{Training der posturalen Kontrolle}

Die Sprunggelenksstrategie sichert die anterior-posteriore Stabilität. Bei einer geringen Verschiebung des Körperschwerpunkts nach hinten werden der Reihenfolge nach der M. tibialis anterior, der Quadrizeps und die Bauchmuskulatur aktiviert (๑ Abb. 2). Bei der Schwerpunktverschiebung nach vorne müssen der M. gastrocnemius, die ischiokrurale Muskulatur und die Rückenstrecker zur Sicherung der posturalen Kontrolle arbeiten. Bei größerer extrinsischer Störung generiert der Körper große rasche Hüftbewegungen unter Aktivierung der Bauchmuskulatur und des M. quadriceps (॰ Abb. 3). Beides, die Sprunggelenks- und die Hüftstrategie, bezeichnet man als „In-Place-Strategien“. Bei In-Place-Strategien ändert sich die Unterstützungsfläche nicht. Anders bei der Schrittstrategie: Hier ist die Krafteinwirkung zu groß oder sie erfolgt zu schnell, so dass ein Ausweichschritt die Unterstützungsfläche vergrößert. Der Massenmittelpunkt kann so über der Unterstützungsfläche gehalten werden (๑ Abb. 4).

Die posturale Kontrolle muss in genau diesen Strategiebereichen trainiert werden. Das Training zur anterior-posterioren Stabilität kann auf einem Schaukelbrett stattfinden. Dort übt der Patient gezielt die Aktivierung der dorsalen bzw. ventralen Bein- und besonders die der sprunggelenksumgebenden Muskulatur. Eine wirksame Sturzprävention kann in Form von Tai-Chi-Übungen stattfinden [39]. Das repetitive Schubsen hat sich bei Patienten mit Parkinson-Syndrom als wirksam gezeigt [18]. Möglicherweise ist diese Therapieform auch bei Patienten nach Schlaganfall im Sinne einer Sturzprävention und zum Mindern der Sturzangst effizient. Entscheidend ist, dass ein Training der posturalen Kontrolle nicht nur statisch, sondern dynamisch und ausreichend intensiv erfolgt. Das Tai Chi betont den dynamischen Aspekt, das repetitive Schubsen zeichnet sich durch eine große Anzahl von Wiederholungen aus. In der Studie zum repetitiven Schubsen erfolgten 180-230 Stöße durch den Therapeu- 


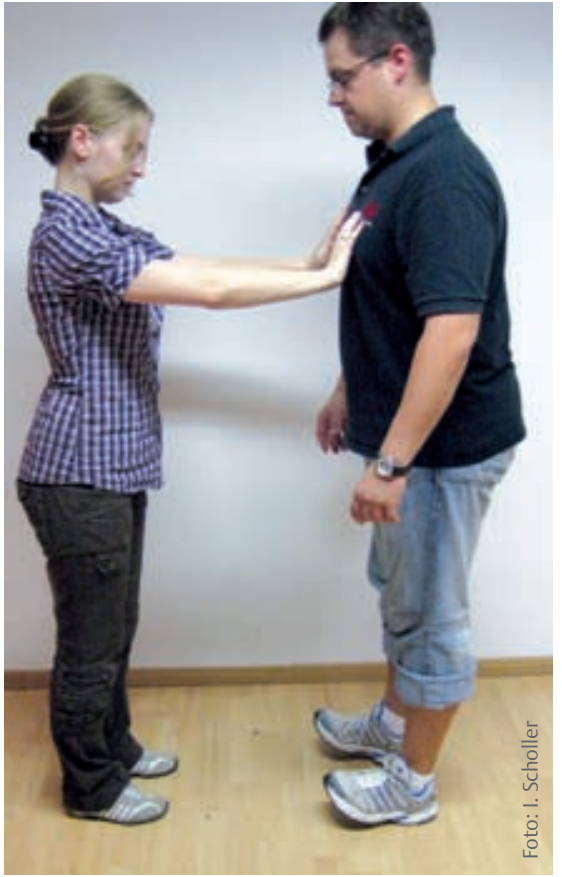

Abb. 2 Sprunggelenksstrategie bei Krafteinwirkung von ventral

ten innerhalb von 20 Minuten. Die Treppe ist neben dem Ausdauer- und Krafttraining auch als Trainingsgerät zur Verbesserung der posturalen Kontrolle geeignet. In der Regel ist ein Geländer vorhanden, das ein Festhalten in kritischen Situationen ermöglicht.

\section{Training der posturalen Kontrolle} unter Dual-Task-Bedingungen

Besondere Anforderungen an die posturale Kontrolle wie das Transportieren von Gegenständen [30, 36] oder Gehen und dabei gleichzeitig den Kopf drehen oder sprechen, ohne die Balance zu verlieren [26], sind ebenfalls Merkmale der außerhäuslichen Gehfähigkeit. Mehrere Aufgaben gleichzeitig zu erledigen, kennzeichnen sogenannte „Dual-Task-Aufgaben“. Eine reduzierte DualTask-Fähigkeit scheint ein Prädiktor für Stürze zu sein. Ein spezifisches Dual-Task-Training kann deshalb helfen, Stürze und Sturzangst zu reduzieren. In frühen Phasen der Rehabilitation oder bei schlechten Aufmerksamkeitsleistungen, wie sie bei rechtshemisphärisch geschädigten Patienten häufig der Fall sind, empfiehlt es sich allerdings, Dual-Task-Aufgaben zunächst nicht zu trainieren. Im Gegenteil: Bei erhöhter Ablenkbarkeit muss der Therapeut mit dem Patienten Strategien erarbeiten, um sich nur auf eine Aktion zu konzentrieren, da sonst die Sturzgefahr zu groß ist.

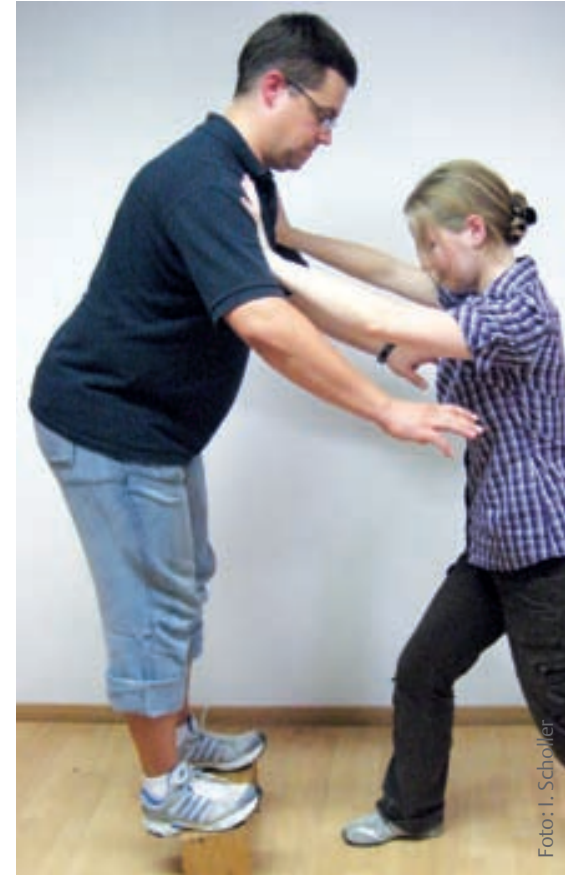

Abb. 3 Hüftstrategie bei Krafteinwirkung von ventral

Training der posturalen Kontrolle -

Terraintraining

2008 publizierten Forscher, dass die Mehrheit der Stürze im häuslichen Umfeld stattfindet [22]. Ein möglicher Zusammenhang besteht mit der Tatsache, dass nur ein Drittel der als gehfähig entlassenen Patienten das Haus verlässt [27] und sich dadurch die Anzahl der außerhäuslichen Stürze reduziert. Das Gehen außerhalb des Hauses erfordert eine sehr gute posturale Kontrolle (॰ Abb. 5).

Viele Patienten haben zudem Angst, auf unebenem Boden oder bei schlechten Wetterverhältnissen zu gehen [36]. Noch größer werden die Anforderungen an die Balancefähigkeit des Patienten beim Fahren mit öffentlichen Verkehrsmitteln oder bei der Lokomotion in belebten Gegenden wie Bahnhöfen oder Kaufhäusern. Die Gefahr für die Betroffenen ist hoch, angerempelt zu werden und zu stürzen. Deshalb wird ein intensives und ausreichend langes Terraintraining in genau diesen Kontexten durchgeführt: Gehen auf unebenem Boden, an der schiefen Ebene ( $\odot$ Abb. 6), im Kies ( $\odot$ Abb. 7), auf Kopfsteinpflaster, im Schnee, bei Regen, in Menschenmengen etc.

\section{Sturztraining}

Obwohl es noch nicht wissenschaftlich belegt ist, zeigt sich das Sturztraining in der

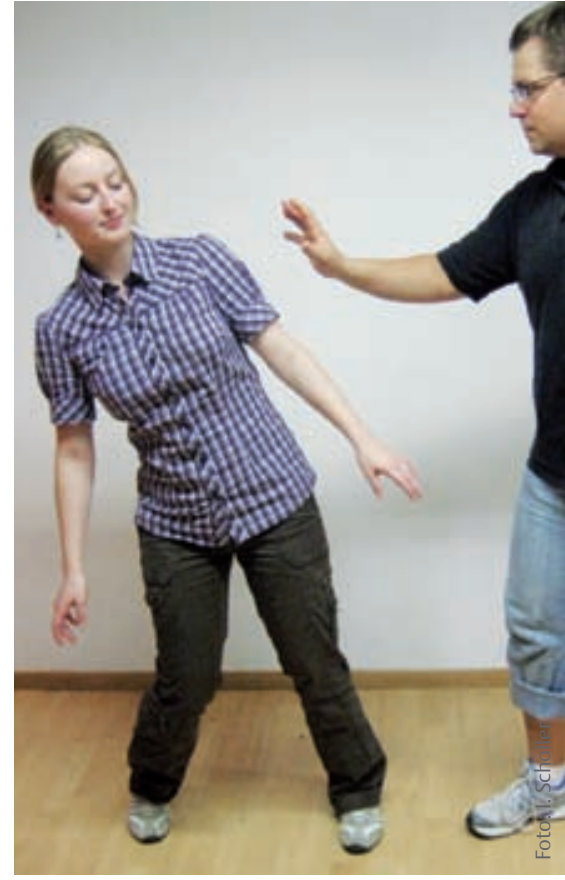

Abb. 4 Schrittstrategie bei Krafteinwirkung von lateral

Therapie als sehr effektiv bei Patienten mit Sturzangst. Das Sturztraining ist eine systematisch aufgebaute Gruppenintervention. Sie besteht aus theoretischen Informationen und praktischen Übungen. Zum Theorieteil gehört die Aufklärung über die häufigsten Frakturen bei Stürzen und über Methoden

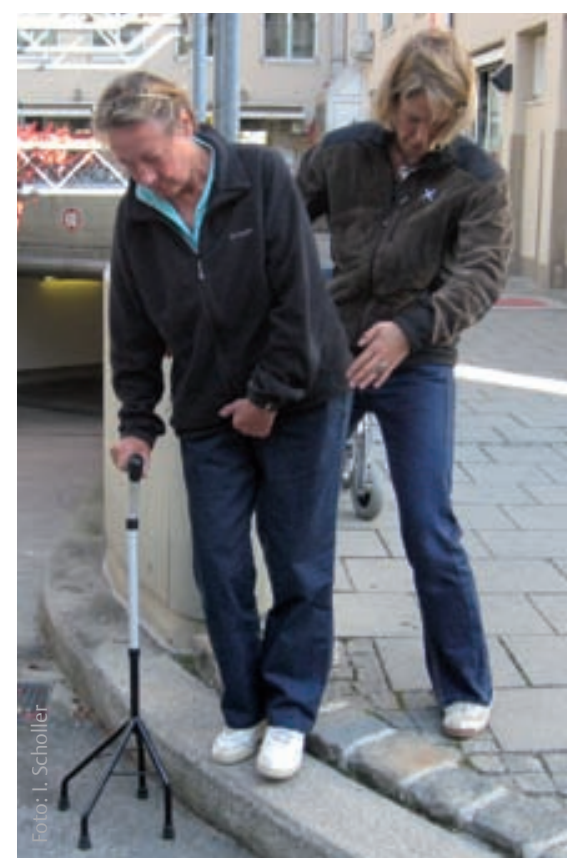

Abb. 5 Bordsteinkanten stellen für die Patienten eine hohe Anforderung an die posturale Kontrolle dar. 


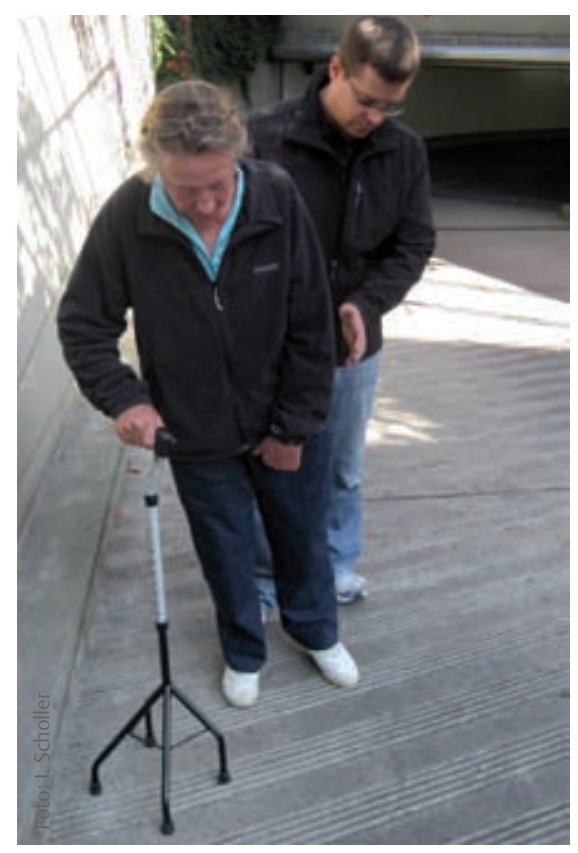

Abb. 6 Terraintraining: schiefe Ebene

des „guten Stürzens“, abgeleitet aus unterschiedlichen Sportarten wie Karate oder Snowboarden. Oberstes Prinzip ist dabei, eine erhöhte Muskelspannung aufzubauen, um die Frakturgefahr zu mindern und eine Verletzung des Kopfes zu vermeiden. Im Praxisteil üben die Patienten verschiedene Strategien des Stürzens, zunächst aus niedriger Höhe auf eine sehr weiche, gepolsterte Unterlage. Systematisch mindert die Therapeutin dann die Sturzhöhe und die Weichheit der Unterlage (๑ Abb. 8).

Zum Praxisteil gehört auch das Üben des Wiederaufstehens. Bei Patienten nach Schlaganfall ist die Angst „nicht wieder hochzukommen und hilflos wie ein Maikäfer auf dem Rücken liegen zu bleiben“

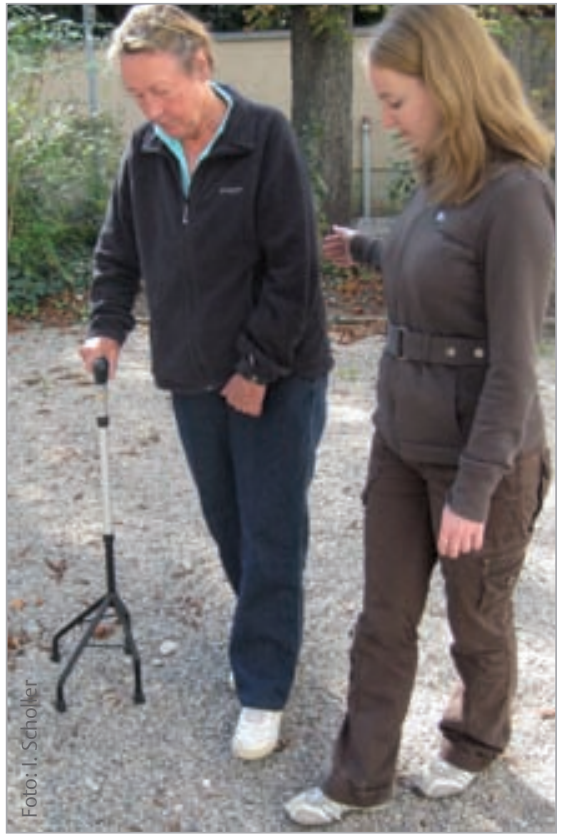

Abb. 7 Terraintraining: Kies

ebenso groß wie die Angst zu stürzen. Diese Angst ist nicht unbegründet, denn laut Forster und Young sind $24 \%$ der gestürzten $\mathrm{Pa}$ tienten nicht in der Lage, selbstständig aufzustehen [9].

\section{Hilfsmittel und Hausbesuch}

Ein Rollator verringert das Sturzrisiko der Patienten [15]. Eine Fußheberorthese mindert bei Schwäche der Dorsalextensoren, die Gefahr hängen zu bleiben und zu stolpern. Diese Hilfsmittel verringern somit auch die Sturzangst der Patienten. Die frühzeitige Versorgung mit Hilfsmitteln fördert das subjektive Sicherheitsgefühl [41]. Die Benutzung von Stöcken durch die Patienten ist häufig unzureichend. Zudem zeigten Stu-

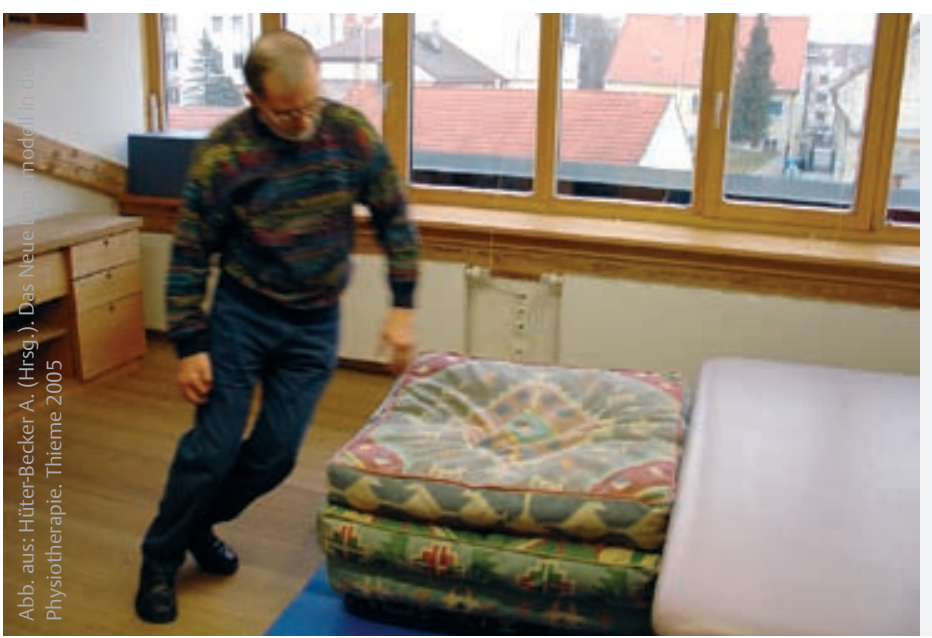

Abb. 8 Sturztraining: praktischer Teil dien, dass die Vermutung, das Verwenden eines Stockes würde die Gewichtsbelastung auf das hemiparetische Bein verringern und zu einer „Verschlechterung des Gangbildes“ führen, unbegründet ist [23, 24]. Durch eine frühzeitige Versorgung mit adäquaten Hilfsmitteln und ein ausreichendes Training mit den Hilfsmitteln im Alltagskontext des Patienten werden die Sturzgefahr und Fallangst gemindert. Bei einem Hausbesuch überprüft die Therapeutin, bis zu welchem Grad der Patient die erreichten Fähigkeiten der Lokomotion und posturalen Kontrolle in sein Lebensumfeld übertragen konnte. Gemeinsam bestimmen sie potenzielle Sturzgefahren wie schlechte Beleuchtung, Türschwellen, Teppichkanten, ungeeignete Aufbewahrungsorte von Alltagsgegenständen (zu hoch/niedrig), das Verwenden von riskanten Steighilfen wie einem Stuhl statt einer Trittleiter und vieles mehr.

\section{Psychotherapeutische Behandlung}

Die Therapie der Angst selbst stützt sich auf verschiedene Störungsmodelle (z.B. lerntheoretische Modelle wie klassische und operante Konditionierung, Modelllernen und auf kognitive Konzepte). Grundlegende Informationen und ein Überblick über die psychologischen Entstehungs- und Behandlungskonzepte von Angststörungen finden sich zum Beispiel bei Kaspar und Möller [21] und bei Margraf [28].

Die psychotherapeutisch-neuropsychologische Therapie von Fallangst orientiert sich hauptsächlich an den Prinzipien der verhaltenstherapeutischen Behandlung pathologischer Angststörungen (z. B. phobischer Störungen, Panikstörung, Agoraphobie). Erste ermutigende Befunde zur integriert-verhaltenstherapeutischen Therapie von Fallangst liegen von Zijlstra vor [46]. Die Verhaltenstherapie bei Angststörungen gilt als das wissenschaftlich am besten fundierte Verfahren, für das sowohl bei der Symptomreduktion als auch bei der Steigerung der Lebensqualität eine hohe Wirksamkeit belegt werden kann [13]. Bei der Fallangst ist jedoch zu beachten, dass es sich hier in der Regel nicht um krankhafte Ängste bzw. eine pathologische Angststörung im Sinne des ICD-10 handelt, sondern die Patienten realistische, wenn auch teilweise übersteigerte Ängste vor dem Fallen zum Beispiel aufgrund eines Sturzes entwickelt haben. Da sich die von Fallangst betroffenen neu- 
rologischen Patienten häufig in höherem Lebensalter befinden, müssen auch gerontologische Konzepte in die Behandlung einbezogen werden [11], wie z.B. das Modell der selektiven Optimierung und Kompensation (SOK-Modell nach Baltes und Carstensen) [3]. Dieses Modell beachtet neurokognitive Einschränkungen, evtl. vorhandene Multimorbidität und begrenzte Ressourcen. Ernst zu nehmen ist auch, dass Fallangst und die Häufigkeit von Stürzen hoch mit Depression korrelieren [4, 22], das heißt, die Behandlung der Fallangst muss die Behandlung der Depression mit einbeziehen. Die Bausteine der kognitiv-verhaltenstherapeutischen Behandlung von Angst werden im Folgenden dargestellt (๑ Kasten „Bausteine der Behandlung von Angst“).

\begin{tabular}{|l|}
\hline $\begin{array}{l}\text { Bausteine der kognitiv-verhaltens- } \\
\text { therapeutischen Behandlung von Angst }\end{array}$ \\
- Psychoedukation \\
- Verhaltens- und Bedingungsanalyse \\
(z.B. Selbstbeobachtungsprotokolle) \\
- Vermittlung von Entspannungstech- \\
niken (PMR nach Jacobson) \\
- praktische Übungen (Exposition im \\
Sinne der systematischen Desensibili- \\
sierung) \\
- gedankliche Übungen bzw. kognitive \\
Umstrukturierung (kognitive Therapie) \\
Die Therapie ist sowohl im Einzel- als auch \\
im Gruppensetting möglich, wobei das \\
Gruppensetting wegen seiner spezifi- \\
schen und unspezifischen Wirkfaktoren \\
vorzuziehen ist.
\end{tabular}

Psychoedukation - Vermittlung von Informationen über Angst und Fallangst Für den Patienten ist es wichtig zu erfahren, dass Angst ein zwar nicht angenehmes, aber normales und notwendiges Gefühl ist. Sie zeigt Bedrohung an und schützt vor Gefahr. Daher muss sie als Alarmsignal ernst genommen werden. Das Verhindern von Stürzen ist wegen der möglichen Konsequenzen sehr wichtig [34]. Ein gewisses Maß an Angst, zum Beispiel in unbekannten Situationen, führt zu einer generellen Aktivierung und ist daher sinnvoll, da sie Aufmerksamkeit erzeugt. Der Körper stellt Energie zur Verfügung und trägt so zur besseren Situationsbewältigung bei [45]. Angst tritt in sehr unterschiedlichem Ausmaß auf und kann Menschen in ihrer Lebensführung erheblich beeinträchtigen. Sie kann aufgrund äußerer Auslöser (z. B. schwer zu überwindende Barriere) und innerer Auslöser (z.B. Angst vor Versagen) auftreten. Meist gibt es nicht nur einen Auslöser, sondern komplexe Bedingungen, die die Angst verursachen. Das Verstehen dieser Zusammenhänge ist für die Patienten bedeutsam. Angstreaktionen sind auf vier Ebenen zu identifizieren:

- körperliche Reaktionen wie Herzklopfen, schnelles Atmen, Schweißausbrüche, Schwindel, Schwächegefühl, Zittern, Unruhe

- emotionale Reaktionen wie starke Angst-, Beklemmungs-, Unsicherheitsgefühle

- kognitive Reaktionen wie „Ich kann das nicht“, „Ich werde das nie schaffen“

- verhaltensmäßige Reaktionen wie sitzen bleiben, die Situation vermeiden bzw. sich zurückziehen

Angst löst Vermeidungsverhalten aus. Dieses Vermeidungsverhalten wird negativ verstärkt durch das Ausbleiben des Gefühls der Angst und so ausgeweitet und generalisiert. Die oben beschriebenen Reaktionen können also bedingen, dass die Angst sich hochschaukelt und ein Teufelskreis mit immer weniger Mut zu Aktivität bei den Patienten einsetzt. Dabei ist zu beachten, dass die Symptome der Angst wie Schwindelgefühle und Stand-/Gangunsicherheit die neurologische Symptomatik noch verstärken. Es ist für den Patienten und den Therapeuten wichtig, die Angst als einen Helfer zu betrachten. Dies ist eine der wichtigsten Voraussetzungen für eine erfolgreiche Behandlung. Allerdings muss auch vermittelt werden, dass es unmöglich ist, Risiken im Leben zu vermeiden und ein gewisses Risiko zu fallen nie ganz ausgeschlossen werden kann.

\section{Patienten sollten darin unterstützt werden, die Angst anzunehmen und sich nicht gegen sie zu stellen, also keine Angst vor der Angst zu entwickeln.}

\section{Exposition und systematische} Desensibilisierung

Zu Beginn der Behandlung erhebt der Therapeut eine sorgfältige Sturzanamnese, um die Vorerfahrungen der Patienten in Bezug auf das Fallen zu erfahren. Ausgehend von dieser Anamnese kann dann im Gespräch eine individuelle Angsthierarchie anhand eines festgelegten Kriteriums (z.B. Anzahl der Hilfsmöglichkeiten in der Nähe, Art der Bodenbeschaffenheit, Zeitdauer) aufgestellt werden.
Die Angsthierarchie besteht aus Abstufungen auf einer Skala von 0 (keine Angst) bis 100 (größte Angst). Durch die Abstufungen der Skala legt der Neuropsychologe mit dem Patienten konkrete Schwierigkeitsstufen fest. Diese werden dann im Sinne der Konfrontationsbehandlung und des erfahrungsorientierten Lernens in den physiotherapeutischen Einzelstunden oder in der interdisziplinären Gruppe nacheinander abgearbeitet und mehrfach wiederholt. Wichtig ist hier, eine ausreichende Differenzierung der Stufen vorzunehmen, damit die jeweils nächste Stufe realisierbar ist. Erschwerende oder erleichternde Bedingungen werden mit den Patienten besprochen. Die Abstufungen der Angsthierarchie repräsentieren demnach die Teilziele des Patienten auf dem Weg zur Gangsicherheit (๑ Kasten „Beispiel für eine Angsthierarchie“ und ๑ Tab. 1).

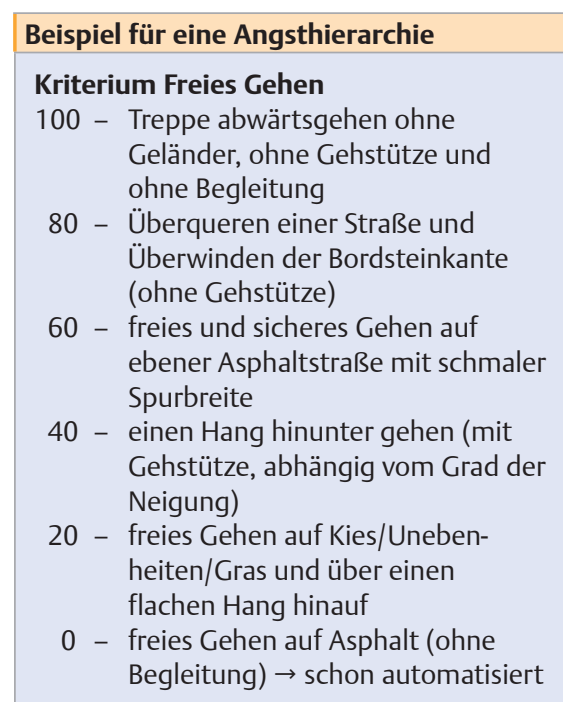

Tab. 1 Alltagsrelevante Bedingungen

\begin{tabular}{|l|l|}
\hline Erschwerende & $\begin{array}{l}\text { Erleichternde } \\
\text { Faktoren }\end{array}$ \\
\hline - unvertraute Strecke & - Begleit-/ \\
- sich nicht festhalten & Hilfsperson \\
können & - etwas zum \\
- Menschenmengen & Festhalten \\
- Bordsteinkanten & - Gehhilfe \\
- Straßenverkehr & \\
- Zeitdruck & \\
- lange Dauer & \\
- ungünstige & \\
Äußerungen und & \\
& Reaktionen von \\
anderen & \\
- Überforderungs- & \\
& \\
& \\
&
\end{tabular}


Die Möglichkeiten der Bewältigung der Angst folgen den Leitsätzen von Wittchen und können als Merkblatt dem Patienten mitgegeben werden (๑ Kasten „Modifiziertes Merkblatt zur Angstbewältigung“ [44]):

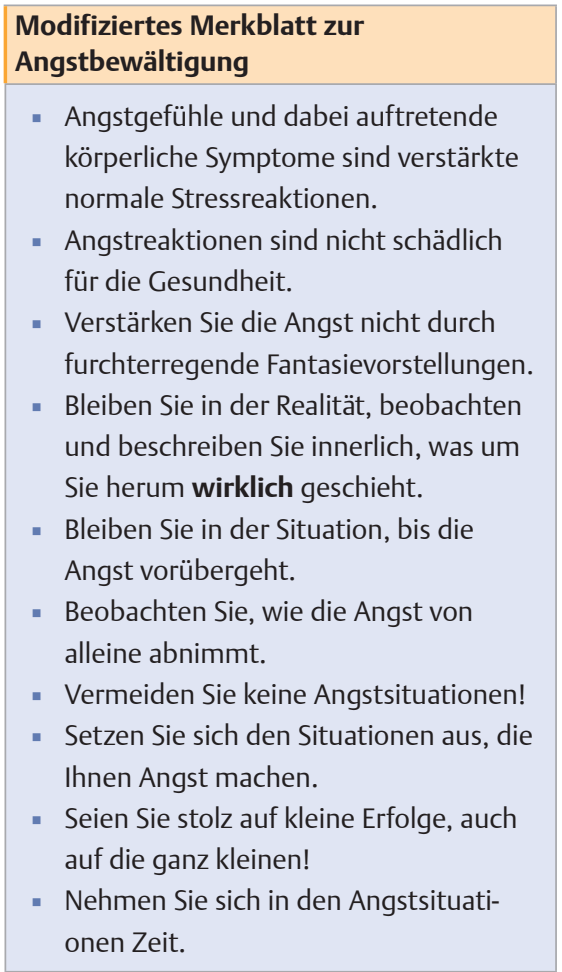

Der Grundsatz also lautet: Angstbesetzte Situationen sollen nicht vermieden werden. Natürlich müssen die zum jeweiligen Zeitpunkt noch notwendigen Hilfestellungen vorhanden sein. Diese dürfen nur stufenweise abgebaut werden. Nur so kann der Angstabbau funktionieren. Wichtig ist es, die Befürchtungen der Patienten ernst zu nehmen und wiederholt zu erfragen, was die Patienten ihrer Meinung nach benötigen, um die Angst zu überwinden.

Die Exposition wird durchgeführt im Sinne einer modifizierten systematischen Desensibilisierung. Dabei ist es wichtig, dass die Exposition „in vivo“, das heißt in der realen Situation, durchgeführt wird. Die Patienten bestimmen selbst das Tempo der Steigerung. Durch die Methode der Progressiven Muskelentspannung lernen sie, die Intensität der Angstgefühle selbst zu beeinflussen. Zudem kann mit den Patienten besprochen werden, was sich ihrer Meinung nach in ihrem Leben verändern wird, wenn sie ihre Ziele in Bezug auf das Gehen erreichen. Hierdurch werden positive Anreize gesetzt und Wunschvorstellungen aktualisiert. Vo-

Tab. 2 Gruppenkonzept „Bewältigung der Fallangst“ 10 Doppelsitzungen à 90 Minuten. Gruppengröße bis zu 8 Teilnehmer

\begin{tabular}{|c|c|}
\hline Baustein & Inhalt \\
\hline Psychoedukation & $\begin{array}{l}\text { Funktion und Auswirkung der Angst } \\
\text { Funktion und Auswirkung der Vermeidung } \\
\text { Informationen über die Behandlung und Bewältigung von Angst }\end{array}$ \\
\hline $\begin{array}{l}\text { Vermittlung einer } \\
\text { Entspannungsmethode }\end{array}$ & PMR (in jeder Sitzung) \\
\hline $\begin{array}{l}\text { Verhaltens- und } \\
\text { Bedingungsanalyse }\end{array}$ & $\begin{array}{l}\text { Erstellen der Angsthierarchie } \\
\text { Erfassung der erschwerenden/erleichternden Bedingungen }\end{array}$ \\
\hline $\begin{array}{l}\text { Kognitive } \\
\text { Verhaltenstherapie }\end{array}$ & $\begin{array}{l}\text { Selbstbeobachtungsprotokoll } \\
\text { Selbstverbalisation } \\
\text { Selbstwertstärkung („Ich-kann-Poster“) }\end{array}$ \\
\hline Expositionsbehandlung & $\begin{array}{l}\text { Systematische Desensibilisierung } \\
\text { Durcharbeiten der Stufen der Angsthierarchie }\end{array}$ \\
\hline Körperliches Training & $\begin{array}{l}\text { Gleichgewichts-, Geschwindigkeits-, Kraft-, Terrain- und Sturz- } \\
\text { training }\end{array}$ \\
\hline $\begin{array}{l}\text { Trainingsprogramm } \\
\text { für zu Hause }\end{array}$ & Aufbau angemessener Aktivitäten (Heimtrainingsprogramm) \\
\hline $\begin{array}{l}\text { Modifikation } \\
\text { der Umwelt }\end{array}$ & $\begin{array}{l}\text { Identifizieren von Gefahrenstellen in der häuslichen und außerhäus- } \\
\text { lichen Umgebung }\end{array}$ \\
\hline
\end{tabular}

raussetzung ist natürlich das Erarbeiten der motorischen Voraussetzungen wie Ausdauer und Muskelkraft für ein sicheres Gehen. Zu Beginn jeder neuen Sitzung wird nach Veränderungen seit der letzten Sitzung, möglichen Ergänzungen auf der Angsthierarchie und evtl. neuen Erkenntnissen gefragt.

\section{Kognitive Umstrukturierung}

Die kognitive Verhaltenstherapie geht davon aus, dass zwischen einer bestimmten Situation und einem Gefühl, welches (vermeintlich) durch die Situation ausgelöst wird, ein kognitiver Bewertungsprozess abläuft. Ziel der kognitiven Umstrukturierung ist es, diese automatischen Gedanken sowie die zugrunde liegenden Faktoren zu bestimmen und gegebenenfalls zu modifizieren. Patienten mit Fallangst zeigen eine hohe Anzahl an dysfunktionalen Überzeugungen, Übergeneralisierungen, voreiligen Schlussfolgerungen, verzerrten Wahrnehmungen und ein unrealistisches negatives Denken. Diese Patienten überschätzen das Risiko zu fallen, sie beurteilen das Fallen als unkontrollierbar und katastrophieren die Folgen eines Sturzes [11]. Derart hinderliche Gedanken sollen identifiziert und ihre negativen Folgen auf Gefühle und Verhalten analysiert werden, um eine Umbewertung angstauslösender Reize zu ermöglichen. Um solche dysfunktionalen Gedanken zu erkennen, kann der Einsatz von Selbstbeobachtungsbögen sehr hilfreich sein [16]. Therapeutisches Ziel ist es, hilfreiche und realistische Gedanken gezielt zu üben und positive Selbstinstruktionen zu implementieren und so selbsthinderliche Gedanken durch selbstermutigende zu ersetzen [8]. Letztlich geht es darum, dass die Patienten wieder Vertrauen in sich und ihre Fähigkeiten bekommen und wieder Sicherheit erreichen. Hier kann zum Beispiel ein „Ich-kann-Poster“ mit einer Aufstellung der vorhandenen Fähigkeiten und Ressourcen gute Dienste leisten.

\section{Interdisziplinäre Gruppentherapie}

Das Ziel der „Sturzangstgruppe“ liegt darin, Angst vor dem Fallen und damit auch vor dem Gehen abzubauen. Erreicht wird dies durch das Zusammenwirken von physiotherapeutischer und verhaltenstherapeutischer Kompetenz in den Bausteinen Theorie und Praxis. Deren Elemente sind weiter oben bereits beschrieben. In der interdisziplinären Zusammenarbeit können die Bausteine jedoch genau aufeinander abgestimmt werden. Die Gruppensituation fördert den Lernerfolg und die Motivation (๑ Tab. 2). 


Autoren
Ingrid Scholler ist Physiotherapeutin, Klinische
Neuropsychologin (GNP) und Psychologische Psy-
chotherapeutin (Verhaltenstherapie). Sie arbeitet
seit 12 Jahren in der Praxis Fries.
Claudia Pott ist seit 1992 Physiotherapeutin und
arbeitet seit 1995 in dem interdisziplinären Team
der Praxis Fries. Außerdem ist sie in der Aus- und
Fortbildung tätig.
Prof. Dr. med. Wolfgang Fries ist Neurologe und
apl. Professor für Medizinische Psychologie und
Neurobiologie an der LMU München. Er leitet seit
1992 die Praxis Prof. Dr. med. Fries für ambulante
neurologische Komplexbehandlung und Nach-
sorge in München.
Ingrid Scholler
Praxis Prof. Dr. med. Fries
Pasinger Bahnhofsplatz 4
81241 München
Tel: 089/896068-0

\section{Literatur}

1. Adkin AL, Frank JS, Carpenter MG et al. Fear of falling modifies anticipatory postural control. Experimental Brain Research 2002; 143: 160-170

2. Andersson AG, Kamwedo K, Applros P. Fear of falling in stroke patients: relationship with previous falls and functional characteristics. Int J Rehabil Res 2008; 31: 261-264

3. Baltes MM, Carstensen LL. Gutes Leben im Alter: Überlegungen zu einem prozessorientierten Metamodell erfolgreichen Alterns. Psychol Rundsch 1996; 47:199-215

4. Belgen B, Beninato M, Sullivan PE et al. The association of balance capacity and falls self-efficacy with history of falling in community-dwelling people with chronic stroke. Arch Phys Med Rehabil 2006; 87: 554-561

5. Berg K, Wood-Dauphinee S, Williams Jl. The Balance Scale: reliability assessment with elderly residents and patients with an acute stroke. Scand J Rehabil Med 1995; 27: 27-36

6. Carpenter MG, Frank JS, Silcher CP et al. The influence of postural threat on the control of upright stance. Experimental Brain Research 2001; 138: 210-218

7. Dias N, Kempen Gl, Todd CJ et al. The German version of the Falls Efficacy Scale-International Version (FES-I). Z Gerontol Geriatr. 2006, 39: 297-300

8. Fliegel S. Selbstverbalisation und Angstbewältigung. In: Linden M, Hautzinger M, Hrsg., Verhaltenstherapie. 3. Aufl. Berlin: Springer; 1996

9. Forster A, Young J. Incidence and consequences of falls due to stroke: a systematic inquiry. BMJ 1995; 311: 83-86

10. Forstmeier S, Maercker A. Psychotherapie im Alter. Psychotherapeutenjournal 2007; 6: 340-352

11. Forstmeier S, Maercker A. Verhaltenstherapie bei Depression, Angst und Trauma folgen im Alter. Psychotherapie im Dialog 2008; 9: 26-32
12. Friedmann SM, Munoz B, West SK et al. Falls and fear of falling: which comes first? A longitudinal prediction model suggests strategies for primary and secondary prevention. J Am Geriatr Soc 2002; 50: 1329-1335

13. Grawe K, Donati R, Bernauer F. Verhaltenstherapie im Wandel: von der Konfession zur Profession. 3. Aufl. Göttingen: Hogrefe; 1994

14. Gulich M, Zeitler H-P. Der Geh-und-Zähl-Test. Dtsch Med Wochenzeitschrift 2000; 125: 245-248

15. Harris JE, Eng J], Marigold DS et al. Relationship of balance and mobility to fall incidence in people with chronic stroke. Phys Ther 2005; 85: 150-158

16. Hautzinger M. Selbstbeobachtung. In: Linden M, Hautzinger M, Hrsg. Verhaltenstherapie. Berlin: Springer; 1996

17. Horak F, Nashner L. Central programming of postural movements: adaptation to altered support configurations. J Neurophysiol 1986; 55: 1369-1381

18. Jöbges M, Heuschkel G, Pretzel C et al. Repetitive training of compensatory steps: a therapeutic approach for postural instability in Parkinson's disease. Neurol Neurosurg Psychiatry 2004; 75: 1682-1687

19. Jørgensen L, Engstad T, Jacobsen BK. Higher Incidence of Falls in Long-Term Stroke Survivors Than in Population Controls. Depressive Symptoms Predict Falls After Stroke. Stroke2002; 33: 542-547

20. Jorstad EC, Hauer K, Becker C et al. Measuring the psychological outcomes of falling: a systematic review. J Am Geriatr Soc 2005; 53: 501-510

21. Kaspar S, Möller H-J, Hrsg. Angst- und Panikerkrankungen. Jena: Gustav Fischer Verlag; 1995

22. Kerse N, Parag V, Feigin VL et al. Falls After Stroke. Results From the Auckland Regional Community Stroke (ARCOS) Study, 2002 to 2003. Stroke 2008; 39: 1890-1893

23. Kuan TS, Tsou JY, Su FC. Hemiplegic gait of stroke patients: the effect of using a cane. Arch Phys Med Rehabil 1999; 80: 777-784

24. Laufer Y. Effects of one-point and four-point canes on balance and weight distribution in patients with hemiparesis. Clin Rehabil 2002; 16: 141-148

25. Laufer Y. The effect of walking aids on balance and weight-bearing patterns of patients with hemiparesis in various stance positions. Phys Ther 2003; 83 : 112-122

26. Lerner-Frankiel MB, Vargas S, Brown MB. Functional community ambulation: what are your criteria? Clinical Management 1990; 6: 12-15

27. Lord SE, McPherson K, McNaughton HK et al. Community ambulation after stroke: how important and obtainable is it and what measures appear predictive? Arch Phys Med Rehabil 2004; 85: 234-239

28. Margraf J, Schneider S. Paniksyndrom und Agoraphobie. In Margraf J, Hrsg. Lehrbuch der Verhaltenstherapie. Band 2: Störungen. Berlin: Springer; 1996

29. Nashner LM, Shupert CL, Horak FB et al. Organization of posture controls: an analysis of sensory and mechanical constraints. Prog Brain Res 1989; 80 411-418

30. Perry J, Garrett M, Gronley JK et al. Classification of Walking Handicap in the stroke Population. Stroke 1995; 26: 982-989

31. Podisadlo D. Richardson S. The timed "Up and Go" test: a test of basic functional mobility for frail elderly persons. J Am Geriatr Soc 1991; 39: 142-148

32. Pohl M, Mehrholz J, Ritschel C et al. Speed-dependent treadmill training in ambulatory hemiparetic stroke patients. Stroke 2002; 33: 553-558

33. Schmid AA, Rittman M. Fear of falling: an emerging issue after stroke. Top Stroke Rehabil 2007; 14: 46-55

34. Schmitt K, Kressig RW. Mobilität und Balance. Therapeutische Umschau 2008; 65: 421-426

35. Shumway-Cook A, Woolacott MH. Motor Control, Translating Research into Clinical Practice. Third edition; Philadelphia: Lippincott Williams and Wilkins; 2004
36. Shumway-Cook A, Patla AE, Stewart A et al. Environmental demands associated with community mobility in older adults with and without mobility disabilities. Phys Ther. 2002 ; 82: 670-681

37. Sibley KM, Carpenter MG, Perry JC et al. Effects of postural anxiety on the soleus H-reflex. Hum Mov Sci 2007; 26: 103-112

38. Syrjälä P, Luukinen H, Pyhtinen J et al. Neurological diseases and accidental falls of the aged. J Neurol 2003; 250: 1063-1069

39. Taylor-Piliae RE, Haskell WL. Tai Chi exercise and stroke rehabilitation. Top Stroke Rehabil 2007; 14: 9-22

40. Tinetti ME, Richman D, Powell L. Falls efficacy as a measure of fear of falling. J Gerontol 1990; 45: 239243

41. Tyson SF, Rogerson L. Assistive walking devices in nonambulant patients undergoing rehabilitation after stroke: the effects on functional mobility, walking impairments, and patients' opinion. Arch Phys Med Rehabil 2009; 90: 475-479

42. Watanabe $Y$. Fear of falling among stroke survivors after discharge from inpatient rehabilitation. Int J Rehabil Res 2005; 28: 149-152

43. Weerdesteyn $\mathrm{V}$, de Niet M, van Duijnhoven $\mathrm{HJ}$ et al. Falls in individuals with stroke. J Rehabil Res Dev 2008; 45: 1195-1213

44. Wittchen H-U et al. Was Sie schon immer über Angst wissen wollten! Basel: Karger; 1994

45. Yerkes RM, Dodson, JD The relation of strength of stimulus to rapidity of habit-formation. Journal of Comparative Neurology and Psychology 1908 18:459-482

46. Zijlstra GAR et al. Interventions to reduce fear of falling in community-living older people: a systematic review. J Am Geriatr Soc 2007; 55: 603-615

47. Zijlstra A, Ufkes T, Skelton DA et al. Do dual tasks have an added value over single tasks for balance assessment in fall prevention programs? A mini-review. Gerontology 2008; 54: 40-49

\section{Bibliografie}

DOI 10.1055/s-0030-1248713

neuroreha 2010; 1: 20-27

(c) Georg Thieme Verlag KG

Stuttgart · New York · ISSN 1611-6496 